Submission ID: 43809

\title{
Mathematical and Physical Simulation of Responses of An Electromagnetic Logging Tool for Studying Macroanizotropy of Sedimentary Deposits
}

M.I. Epov (IPGG SB RAS, NSU, NSTU), V.N. Glinskikh* (IPGG SB RAS, NSU, NSTU), V.N. Eremin (SPEGE "Looch"), M.N. Nikitenko (IPGG SB RAS), A.N. Petrov (SPEGE "Looch"), I.V. Surodina (IPGG, ICMMG SB RAS), I.V. Mikhaylov (IPGG SB RAS, NSU)

\section{SUMMARY}

The presented work is devoted to development of an electromagnetic tool designed to study electrophysical parameters of sedimentary rocks characterized by macroanisotropic properties. Based on new theoretical and engineering ideas, we have proposed, designed and developed an electromagnetic tool with a high spatial resolution. We have developed complexes of algorithms and computer programs for the analysis of the new electromagnetic logging tool signals in spatially inhomogeneous media. The measured electromagnetic signals and their sensitivity functions to electrophysical parameters of the models are studied. With respect to processing and interpretation of the electromagnetic tool data, we have developed algorithms for filtering, transforming signals into apparent parameters, detecting formation boundaries, and numerical inversion aimed at the determination of the parameters of an isotropic and anisotropic formation, taking into consideration the host medium. We provide examples of the test results obtained in a metrological tank with electrolyte, as well as in natural and artificial freshwater reservoirs. A prototype of the electromagnetic tool was designed and created, with further testing of the prototype and its main components on laboratory test facilities and in conditions close to those in boreholes. 
Математическое и физическое моделирование сигналов электромагнитного зонда для изучения макроанизотропии осадочных отложений

М.И. ЭПов (ИНГГ СО РАН, НГУ, НГТУ), В.Н. Глинских* (ИНГГ СО РАН, НГУ, НГТУ), В.Н. Еремин (НПП ГА «Луч»), М.Н. Никитенко (ИНГГ СО РАН), А.Н. Петров (НПП ГА «Луч»), И.В. Суродина (ИНГГ, ИВМиМГ СО РАН), И.В. Михайлов (ИНГГ СО РАН, НГУ)

\section{Введение}

Вовлечение в изучение и разработку новых нефтегазовых залежей приводит к расширению круга задач промысловой геофизики. Одна из них связана с нефтенасыщенными пластамиколлекторами, которые представлены тонким переслаиванием песчаных и глинистых прослоев. Как известно, тонкая слоистость осадочных отложений наблюдается не только в сантиметровом, но и метровом масштабах, что обусловлено условиями осадконакопления и осадочным материалом. Разрабатывается много промышленных нефтеносных горизонтов, характеризующихся тонкослоистостью, но существуют трудности при их выделении и количественной оценки параметров, в первую очередь петрофизических.

Одной из основных физических характеристик терригенных пород, по которой определяется тип флюидонасыщения, является удельное электрическое сопротивление (УЭС). Тонкослоистые коллектора, представленные чередованием прослоев глин и нефтенасыщенных песчаников, характеризуются низкими значениями УЭС. Эти значения такие, что при традиционной петрофизической интерпретации указывают на водо-, а не нефтенасыщение. Основной причиной низкого УЭС является содержание тонких проводящих прослоев глин.

Представленная работа посвящена разработке новых приборов для электромагнитного каротажа в нефтегазовых скважинах. Целью работы является создание скважинного электромагнитного зонда, предназначенного для изучения электрофизических параметров осадочных пород, характеризующихся макроанизотропными свойствами.

\section{Электрическая макроанизотропия осадочных пород}

Традиционно информация об УЭС извлекается из данных методов постоянного (БК, БКЗ) и переменного (ИК, ВИКИЗ) тока. В работе [Рытов, 1955] показано, что электрическое и электромагнитные поля в тонкослоистой среде эквивалентны их значениям в однородной макроанизотропной при условии, что расстояние между источником и точкой измерения существенно превосходит мощность прослоев. Такая среда является трансверсальноизотропной, т.е. её УЭС в горизонтальной плоскости и вертикальном направлении различаются. В силу известного парадокса анизотропии сигнал, измеренный в зондах постоянного тока, определяется только горизонтальным УЭС и практически не зависит от вертикального. При субвертикальном пересечении скважиной тонкослоистого коллектора измеренный индукционный сигнал зависит только от горизонтального УЭС.

Решение задачи определения параметров отдельных прослоев тонкослоистого коллектора в общей постановке не представляется возможным в силу их большого числа и относительно малой мощности. Поэтому разделение по типу флюидонасыщения проводят, определяя значения горизонтального и вертикального УЭС или коэффициент электрической анизотропии [Табаровский и др., 1976]. Значения последнего, превышающие единицу, будут указывать на наличие в коллекторе тонких глинистых прослоев. Известны другие подходы, позволяющие свести рассматриваемую задачу к определению средних параметров прослоев без введения макроанизотропной модели [Глинских, Эпов, 2009а, 2009б].

\section{Изучение макроанизотропии с использованием электромагнитных зондов}

Традиционно задачу определения параметров макроанизотропного коллектора решают, применяя зонды с многокомпонентными измерениями, где генерация токов и измерения э.д.с. выполняется в ортогональных катушках. Такие комплексы используются ведущими зарубежными компаниями Backer Hughes, Halliburton, Schlumberger, Weatherford. По этой теме опубликовано большое количество работ, обзор которых мог бы составить отдельную книгу. 
Для изучения электрической макроанизотропии необходима система «возбуждениянаблюдения», электромагнитные отклики которой зависят как от горизонтального, так и вертикального УЭС. Такая возможна с применением соосно расположенных катушек, представленных в виде замкнутого соленоида (тороидальная катушка). Рядом исследователей рассматривалось применение тороидальных катушек для изучения УЭС горных пород и электрической анизотропии применительно к каротажу, в том числе в процессе бурения.

Один из первых результатов применения каротажных зондов с тороидальными катушками опубликован в работе [Arps, 1967]. В предложенной конфигурации используются две генераторные катушки, симметрично расположенных на корпусе прибора. Использование тороидальных катушек в зондах для каротажа в процессе бурения предложено в [Redwine, Osborn, 1968]. Другое их применение связано с азимутальными измерениями с помощью набора кнопочных электродов, находящихся на корпусе прибора [Clark et al, 1993]. Известны и более поздние работы, посвященные применению тороидальных катушек в электромагнитных зондах [Gianzero, Bittar, 2007]. Однако упоминания о практической реализации таких приборов неизвестно. В работе [Аксельрод, 2012] описано использование тороидальных катушек в варианте наддолотного каротажа и другие примеры.

Наряду с предлагаемыми техническими решениями выполнен значительный объём численного моделирования электромагнитных полей, возбуждаемых тороидальными катушками. В работе коллег [Могилатов, Борисов, 2003] получены решения задачи об электромагнитном поле магнитного кругового тока в слоисто-однородных моделях сред. Известны работы [Каринский, 2003], в которых выполнено численное моделирование электромагнитных откликов в осесимметричных анизотропных моделях сред на основе метода конечных разностей, а также в трехмерных моделях с использованием метода конечных элементов.

Необходимо отметить, что в сильнонаклонных и горизонтальных скважинах возможно изучение электрической макроанизотропии с использованием электромагнитных зондов, имеющих традиционные соосные индуктивные катушки [Эпов и др., 2014, 2015, 2016].

\section{Многокатушечный многочастотный многорежимный электромагнитный зонд}

На основе новых теоретических и инженерно-технических идей предложен, спроектирован и разработан электромагнитный зонд, обладающий высоким пространственным разрешением [Эпов и др., 2014а, 2014б]. Основные решаемые задачи связаны с изучением его возможностей на основе масштабного математического и физического моделирования.

Многокатушечный многочастотный электромагнитный зонд состоит из немагнитного металлического корпуса и расположенных на нём нескольких тороидальных генераторных и приёмных катушек. Операционные частоты имеют килогерцовый диапазон. Выполняются измерения реальной и мнимой частей э.д.с. (или амплитудно-фазовые измерения). Предложена оригинальная система «возбуждения-наблюдения» в нескольких режимах.

Выполнено обоснование оптимальной конфигурации прибора на основе численного моделирования электромагнитных сигналов зондов. Для этого разработана программноалгоритмическая база решения прямых задач в слоисто-однородных и вертикальнонеоднородных осесимметричных изотропных и анизотропных моделях сред. Выполнено численное моделирование электромагнитных сигналов в моделях, которые позволяют описывать прибор, скважину, сложную прискважинную зону, состоящую из неограниченного числа цилиндрических зон, а также набор пластов с горизонтальными границами.

Для модели среды «скважина - пласт» с учётом корпуса прибора построены пространственные распределения источников измеряемых сигналов - вихревых токов в пласте. Показано, что вихревые токи имеют горизонтальную и вертикальную составляющие и измеряемые сигналы зависят от горизонтального и вертикального УЭС пласта. Установлена однозначная связь измеряемых сигналов с УЭС пласта и электрической анизотропией и показано, что сигналы имеют высокий уровень, значительный динамический диапазон и независимы. Сделан вывод о том, что влияние УЭС бурового раствора и радиуса скважины незначительно.

Проведено масштабное моделирование сигналов и функций чувствительностей в изотропных и анизотропных моделях «скважина - зона проникновения - пласт» с учётом корпуса прибора. Чувствительность сигнала к параметру описывает отношение погрешности измерения к 
погрешности определения параметра. Чем выше чувствительность, тем достовернее будет определяться модельный параметр. Показано, что чувствительности измеряемых сигналов высокие к УЭС зоны проникновения и пласта, а также значительные к коэффициенту электрической анизотропии. При этом отмечается низкая чувствительность к УЭС бурового раствора и радиусу скважины. Проведенные исследования позволили сделать выбор оптимальной конфигурации зонда: определить количество генераторных и приёмных катушек, операционные частоты, длины зондов, а также типы измеряемых сигналов.

Для выбранной конфигурации зонда проведено численное моделирование и сравнительный анализ измеряемых сигналов в типичных осесимметричных моделях изотропных и анизотропных пластов-коллекторов ограниченной мощности. Реалистичное моделирование проведено с учётом конечных размеров зонда на основе методов конечных разностей и элементов. Исследовано поведение электромагнитных сигналов в зависимости от параметров скважины, зоны проникновения и пласта, а также вмещающей среды. Показаны возможности изучения макроанизотропных свойств горных пород с использованием предложенного электромагнитного зонда с тороидальными катушками.

Применительно к обработке и интерпретации данных электромагнитного зонда разработаны алгоритмы фильтрации, трансформации сигналов в кажущиеся УЭС и коэффициент электрической анизотропии, выделения контрастных геоэлектрических границ, численной инверсии с определением параметров изотропного и анизотропного пласта, в том числе с учётом вмещающей среды. Выполнено тестирование алгоритмов на зашумлённых синтетических данных и показана их высокая эффективность. Разработанные алгоритмы составляют основу разрабатываемой компьютерной интерпретационной системы.

На основе результатов математического моделирования создан лабораторный макет электромагнитного зонда и проведено его всестороннее тестирование. Показана высокая степень согласования результатов математического и физического моделирования, проведенного в метрологическом баке с электролитом, а также в естественных и искусственных водоемах. В результате исследований спроектирован и разработан опытный образец электромагнитного зонда, использующий наборы генераторных и приёмных тороидальных катушек, работающий на нескольких операционных частотах и реализующий ряд режимов его работы [Эпов и др., 2016а, 2016б, 2016в, 2016г]. Проведены испытания на лабораторных стендах и в реальных скважинных условиях. Внешний вид электромагнитного зонда с модулем телеметрии приведен на рис. 1.

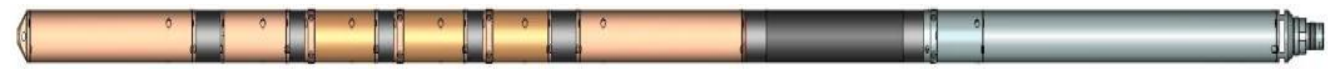

Рисунок 1 Внешний вид электромагнитного зонда.

\section{Выводы}

Разработаны комплексы алгоритмов и программ для моделирования электромагнитных сигналов нового электромагнитного зонда в пространственно неоднородных моделях сред. Изучены измеряемые сигналы и их функции чувствительностей к электрофизическим параметрам моделей и сделаны выводы о высоком пространственном разрешении. Выбрана оптимальная конфигурация зондовой системы, включая длины зондов, операционные частоты, типы измеряемых сигналов и режимы работы. Проведено масштабное математическое моделирование сигналов электромагнитного зонда и выполнен комплексный анализ в типичных моделях нефтегазовых коллекторов с учётом реалистичного описания зондовой системы. Создан лабораторный макет электромагнитного зонда и проведен сопоставительный анализ результатов математического и физического моделирования. Результаты тестирования в метрологическом баке с электролитом, а также в естественных и искусственных пресноводных водоёмах, показали высокую степень соответствия. Спроектирован и создан опытный образец электромагнитного зонда. Проведено тестирование опытного образца и его основных узлов на лабораторных стендах и в условиях, приближенным к скважинным, а также выполнены успешные опытно-промышленные испытания. 


\section{Библиография}

Аксельрод, С.М. [2012] Методы опережающей навигации при бурении горизонтальных скважин. Каротажник, 219, с. 87-122.

Глинских, В.Н., Эпов, М.И. [2009] Численное моделирование диаграмм электромагнитного каротажа при описании электропроводности тонкослоистых коллекторов. Геология $u$ геофизика, 50(8), с. 941-949.

Глинских, В.Н., Эпов, М.И. [2009] Новый подход к моделированию и инверсии данных электромагнитного каротажа в тонкослоистых коллекторах. Геофизический журнал, 31(4), c. 119-127.

Каринский, А.Д. [2003] Решения прямых задач о поле тороидальной антенны в анизотропной среде. Физика Земли, 1,с. 9-20.

Каюров, К.Н., Еремин, В.Н., Эпов, М.И., Глинских, В.Н., Сухорукова, К.В., Никитенко, М.Н. [2014] Аппаратура и интерпретационная база электромагнитного каротажа в процессе бурения. Нефтяное хозяйство, 12, с. 112-115.

Могилатов, В.С., Борисов, Г.А. [2003] Возбуждение слоистых геоэлектрических сред гармоническим магнитным током. Сибирский журнал индустриальной математики, 6(1), c. 77-87.

Рытов, С.М. [1955] Электромагнитные свойства слоистой среды. ЖЭТФ, 29(5), с. 605-616.

Табаровский, Л.А., Каганский, А.М., Эпов, М.И. [1976] Электромагнитное поле гармоническго источника в анизотропной слоистой среде. Геология и геофизика, 17(3), с. 94-99.

Эпов, М.И., Глинских, В.Н., Сухорукова, К.В., Никитенко, М.Н., Еремин, В.Н. [2015] Численное моделирование и инверсия данных электромагнитного каротажа в процессе бурения и шаблонирования нефтегазовых скважин. Геология и геофизика, 56(8), 1520-1529.

Эпов, М.И., Никитенко, М.Н., Глинских, В.Н., Еремин, В.Н. [2016] Изучение электрической макроанизотропии интервалов наклонно-горизонтальных скважин по данным высокочастотного индукционного каротажа в процессе бурения. Каротажник, 269, с. 94-109.

Эпов, М.И., Глинских, В.Н., Никитенко, М.Н. [2014] Способ измерения удельной электропроводности и электрической макроанизотропии горных пород. Патент на изобретение RU 2525149.

Эпов, М.И., Еремин, В.Н., Манштейн, А.К., Петров, А.Н., Глинских, В.Н. [2014] Устройство для измерения удельной электропроводности и электрической макроанизотропии горных пород. Патент на изобретение RU 2528276.

Эпов, М.И., Еремин, В.Н., Петров, А.Н., Глинских, В.Н. [2016] Электромагнитный зонд для каротажа в нефтегазовых скважинах. Патент на изобретение RU 2583867.

Эпов, М.И., Еремин, В.Н., Петров, А.Н., Глинских, В.Н., Суродина, И.В., Киселев, В.В., Никитенко, М.Н. [2016] Устройство для генерации электромагнитного поля тороидальной катушкой в геологической среде. Патент на изобретение RU 2579177.

Эпов, М.И., Еремин, В.Н., Петров, А.Н., Глинских, В.Н., Суродина, И.В., Киселев, В.В. [2016] Устройство для регистрации характеристик электромагнитного поля с использованием тороидальных катушек. Патент на изобретение RU 2578774.

Эпов, М.И., Еремин, В.Н., Петров, А.Н., Глинских, В.Н. [2016] Электромагнитный зонд для каротажа в нефтегазовых скважинах. Патент на промышленный образец RU 97539.

Arps, J.J. [1967] Inductive resistivity guard logging apparatus including toroidal coils mounted on a conductive stem. US Patent 3,305,771.

Clark, B., Bonner, S.D., Jundt, J.A., Luling, M.G. [1993] Well logging apparatus having toroidal induction antenna for measuring, while drilling, resistivity of earth formation. US Patent 5,235,285.

Gianzero, S., Bittar, M. [2007] Determining formation anisotropy based in part on lateral current flow measurements. US Patent 7,227,363.

Redwine, F.H., Osborn, W.F. [1968] Formation resistivity measurement while drilling utilizing physical conditions representative of the signals from a toroidal coil located adjacent the drilling bit. US Patent 3,408,561. 


\section{References}

Akselrod, S.M. [2012] Advanced geosteering methods when drilling horizontal wells. Karotazhnik, 219, p. 87-122.

Glinskikh, V.N., Epov, M.I. [2009] Conductivity of layered reservoirs in induction data processing: continuous-function approximation. Russian Geology and Geophysics, 50(8), p. 720-725.

Glinskikh, V.N., Epov, M.I. [2009] A new approach to simulation and inversion of electromagnetic logs in thinly bedded reservoirs. Geophysical Journal, 31(4), p. 119-127.

Karinsky, A.D. [2003] Solutions of direct problems for the field of a toroidal antenna in an anisotropic medium. Physics of the Earth, 1, p. 9-20.

Kayurov, K.N., Eremin, V.N., Epov, M.I., Glinskikh, V.N., Sukhorukova, K.V., Nikitenko, M.N. [2014] Electromagnetic-logging-while-drilling equipment and numerical inversion software. Oil industry, 12, p. 112-115.

Mogilatov, V.S., Borisov, G.A. [2003] Excitation of layered geoelectrical media by a harmonic magnetic current. Siberian Journal of Industrial Mathematics, 6(1), p. 77-87.

Rytov, S.M. [1955] Electromagnetic Properties of a layered medium. Journal of experimental and theoretical physics, 29(5), p. 605-616.

Tabarovsky, L.A., Kagansky, A.M., Epov, M.I. [1976] Electromagnetic field of a harmonic source in an anisotropic layered medium. Geology and Geophysics, 17(3), p. 94-99.

Epov, M.I., Glinskikh, V.N., Sukhorukova, K.V., Nikitenko, M.N., Eremin, V.N. [2015] Forward modeling and inversion of LWD induction data. Russian Geology and Geophysics, 2015, 56(8), p. 1194-1200.

Epov, M.I., Nikitenko, M.N., Glinskikh, V.N., Eremin, V.N. [2016] Studying the electric macroanisotropy in deviated and horizontal boreholes according to high-frequency induction logging while drilling data. Karotazhnik, 269, p. 94-109.

Epov, M.I., Glinskikh, V.N., Nikitenko, M.N. [2014] Method for measuring the specific electric conductivity and electrical macroanisotropy of rocks. Invention patent RU 2525149.

Epov, M.I., Eremin, V.N., Manstein, A.K., Petrov, A.N., Glinskikh, V.N. [2014] Device for measuring the specific electrical conductivity and electrical macroanisotropy of rocks. Invention patent RU 2528276.

Epov, M.I., Eremin, V.N., Petrov, A.N., Glinskikh, V.N. [2016] Electromagnetic tool for logging in oil and gas wells. Invention patent RU 2583867.

Epov, M.I., Eremin, V.N., Petrov, A.N., Glinskikh, V.N., Surodina, I.V., Kiselev, V.V., Nikitenko, M.N. [2016] Device for generating an electromagnetic field by a toroidal coil in a geological environment. Invention patent RU 2579177.

Epov, M.I., Eremin, V.N., Petrov, A.N., Glinskikh, V.N., Surodina, I.V., Kiselev, V.V. [2016] Device for recording characteristics of an electromagnetic field using toroidal coils. Invention patent RU 2578774.

Epov, M.I., Eremin, V.N., Petrov, A.N., Glinskikh, V.N. [2016] Electromagnetic tool for logging in oil and gas wells. Patent for industrial design RU 97539.

Arps, J.J. [1967] Inductive resistivity guard logging apparatus including toroidal coils mounted on a conductive stem. US Patent 3,305,771.

Clark, B., Bonner, S.D., Jundt, J.A., Luling, M.G. [1993] Well logging apparatus having toroidal induction antenna for measuring, while drilling, resistivity of earth formation. US Patent 5,235,285.

Gianzero, S., Bittar, M. [2007] Determining formation anisotropy based in part on lateral current flow measurements. US Patent 7,227,363.

Redwine, F.H., Osborn, W.F. [1968] Formation resistivity measurement while drilling utilizing physical conditions representative of the signals from a toroidal coil located adjacent the drilling bit. US Patent 3,408,561. 